\title{
Proposta e Simulação De um Sistema de Controle de Motor CC Para Veículos Elétricos Con- SIDERANDO A DiNÂMICA VEICULAR
}

\author{
Paulo R. U. Guazzelli ${ }^{1}$, Marcelo Suetake ${ }^{1}$, Carlos A. De Francisco ${ }^{1}$, Osmar Ogashawara ${ }^{1}$.
}

1. Departamento de Engenharia Elétrica - DEE, Centro de Ciências Exatas e de Tecnologia - CCET, Universidade Federal de São Carlos - UFSCar

Rodovia Washington Luís - km 235, SP - 310, CEP 13565-905, São Carlos, SP, Brasil

E-mails: prubaldo92@hotmail.com, mclsuetake@gmail.com, engcar-

losehotmail.com, osmarogaeufscar.br

\begin{abstract}
In this paper, a DC motor speed controller is presented for application in electric vehicles. The controller limits the motor's maximum armature current for protection purposes and allow regenerative braking. An electric vehicle simulation is presented in order to evaluate the proposed controller.
\end{abstract}

Keywords_Control, Electric Vehicles, Regeneration, Simulation, Dynamics.

Resumo - Neste artigo é apresentado um controlador de velocidade para uso em veículos elétricos. O controlador limita a máxima corrente da armadura da máquina elétrica, para sua proteção, e possibilita frenagem regenerativa. Uma simulação de um veículo elétrico é apresentada, para avaliação do controlador proposto.

Palavras-chave—Controle, Veículos Elétricos, Regeneração, Simulação, Dinâmica.

\section{Introdução}

Os veículos elétricos (EV) e veículos elétricos híbridos (HEV) são algumas das alternativas para a diminuição da emissão de $\mathrm{CO} 2$ em $50 \%$ até o ano de 2050, conforme indicado por IEA (2009). As grandes montadoras de veículos possuem, no exterior, automóveis elétricos sendo produzidos em escala comercial. No Brasil, a Fiat, em parceria com a Hidrelétrica Itaipu, desenvolveu um veiculo elétrico do modelo Palio e estão realizando testes, conforme Itaipu (2004). O desenvolvimento desta tecnologia é importante para a indústria automobilística nacional. Este artigo apresenta a modelagem, simulação e controle de um motor CC para aplicação em veículos elétricos.

Na seção 2 apresentam-se os objetivos da pesquisa, na seção 3 detalha-se o modelo desenvolvido e o controle do motor, na seção 4 apresentam-se os resultados simulados e na seção 5 são feitas as conclusões.

\section{Propósitos}

Dentro da visão do Grupo de pesquisa em Veículos Elétricos da UFSCar de construir um veículo elétrico, desenvolveu-se uma plataforma de simulação (Guazzelli, De Francisco e Ogashawara, 2013), conforme é mostrado na Figura 1, que modela os subsistemas usualmente encontrados em um veículo elétrico, utilizando-se as ferramentas do software MatLab: Simulink, SimPowerSystems e SimDriveline. Essas ferramentas já foram utilizados para simular veículos elétricos movidos a célula de combustível, como o Honda FCX Clarity em Njoya, Tremblay and
Dessaint, (2009), e veículos híbridos, como o Toyota Prius, Sefyk and Hyiama, (2011). Veículos puramente elétricos movidos a bateria (BEV) como o Nissan Leaf e o Tesla Roadster foram simulados no software EXCEL (Ambos em Hayes et al., 2011). Neste trabalho, os softwares citados do MatLab serão usados para simular um BEV.
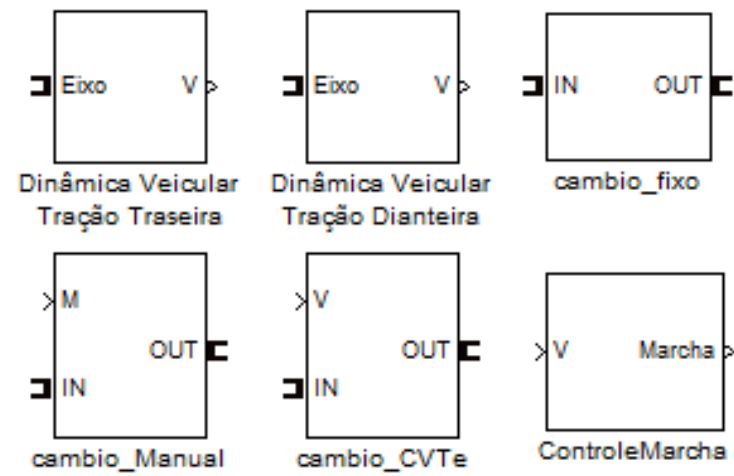

Traçăo Dianteira
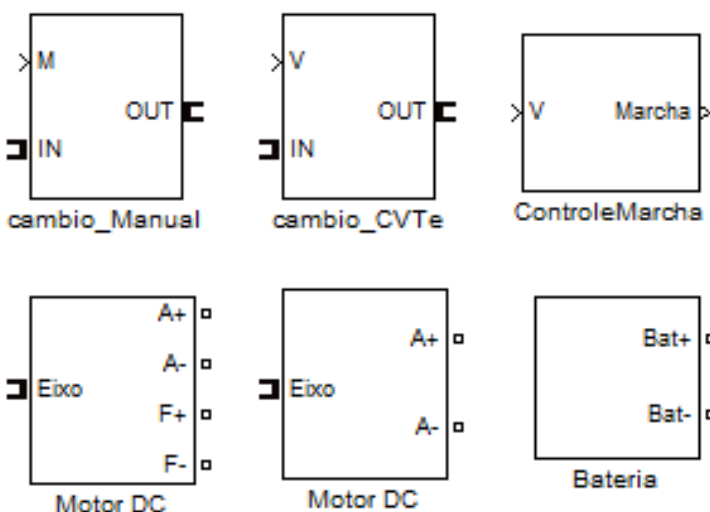

Bobinado
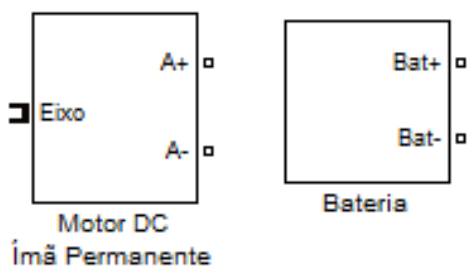

Bateria
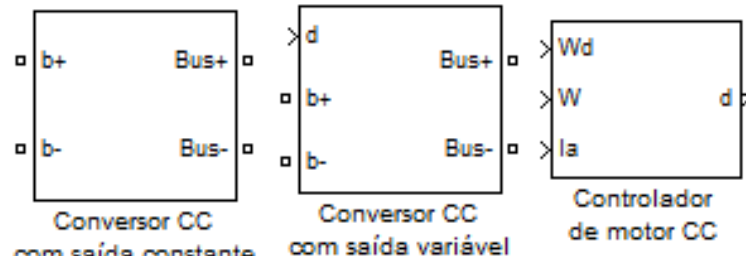

com saida constante de motor $\mathrm{CC}$

Figura 1. Biblioteca de simulação desenvolvida. 
Diversas máquinas elétricas podem ser usadas para mover um veículo elétrico. O Nissan Leaf utiliza um motor síncrono de ímã permanente, conforme Nissan (2011). No entanto, a utilização deste motor contém um revés considerável. Para sua fabricação, é necessário metais de terras raras, como o Neodímio cuja disponibilidade é limitada. Assim, sua fabricação em escala global fica prejudicada. Máquinas elétricas que não precisam de ímãs permanentes estão sendo usadas nos veículos elétricos como alternativa a este problema. Por exemplo, o Tesla Model S, conforme Tesla (2013), e o Fiat Palio da Hidrelétrica de Itaipu, conforme Itaipu (2004), utilizam motor de indução, que não necessita de metais de terras raras.

Outra máquina elétrica que não contém metais de terras raras é o motor de corrente contínua com enrolamento de campo. Assim, ele também se constitui em uma alternativa a ser analisada. A seu favor, conta a sua facilidade de controle frente ao motor de indução. Por exemplo, seu controle já foi estudado em Hearn et al., (2010), com o intuito de utilizá-lo em um veículo automotor.

A partir do controlador proposto, também são estudadas técnicas de utilização de frenagem regenerativa do motor, de modo que possam ser analisados os seus benefícios em um determinado veículo.

\section{Métodos}

\subsection{Parte Elétrica do Veículo}

Para funcionamento do controlador, faz-se necessário o desenvolvimento de um bloco capaz de modelar um conversor CC com saída variável (Figura 2).

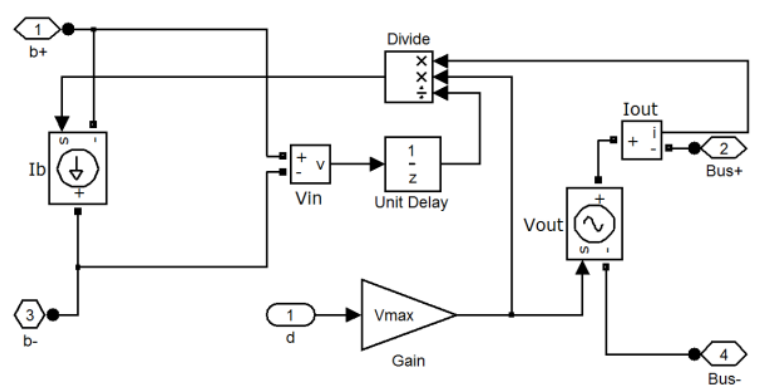

Figura 2. Conversor CC com modulação.

Este bloco simula um atuador que permite que se varie a tensão sobre o motor, possibilitando o seu controle. A partir do ciclo de trabalho desejado, ele produz na saída uma tensão correspondente, entre 0 e uma tensão máxima. Ele mede a corrente neste barramento de saída (onde está o motor) e a tensão no barramento de entrada (onde se encontra o banco de baterias). Baseado na conservação de energia, ele determina qual é a corrente que circula pelas baterias.

Para a simulação de um circuito PWM faz-se necessário um intervalo de amostragem suficiente- mente pequeno para a discretização das formas de onda envolvidas. No intuito de se contornar este problema, foi implementado um bloco baseado num modelo de valores médios. Isto possibilitou um ganho de eficiência computacional sem comprometer os resultados obtidos.

\subsection{Parte Mecânica do Veículo}

A simulação da parte mecânica do veículo foi realizada considerando-se dois diferentes blocos de transmissão. Primeiramente, escolheu-se um bloco de transmissão fixa (Figura 3):

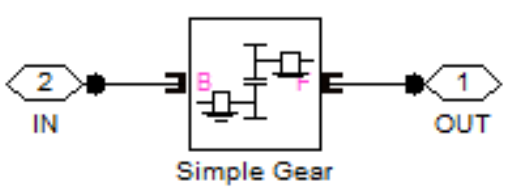

Figura 3. Transmissão fixa.

A transmissão fixa é uma redução, que é o análogo mecânico de um transformador elétrico. A velocidade do eixo de saída é igual à velocidade do eixo de entrada dividida por um valor fixo, e o torque do eixo de saída é igual ao torque de entrada multiplicado pelo mesmo valor fixo. Assim, a potência nos dois eixos é igual.

A outra transmissão escolhida foi uma caixa manual de marchas (Figura 4):

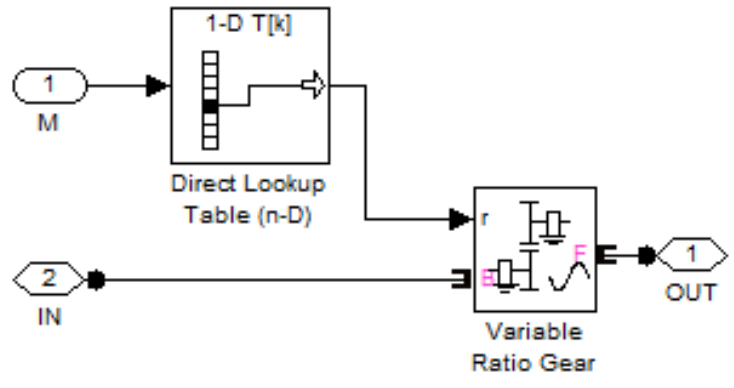

Figura 4. Transmissão manual de $\mathrm{N}$ marchas.

A transmissão manual consiste de um conjunto finito de reduções, em que se seleciona qual delas está acoplando o motor ao veículo em um determinado momento. Isso é possível por meio do bloco $D i$ rect Lookup Table, no qual são programados os valores das reduções de cada marcha, e do bloco Variable Ratio Gear, que é uma redução cujo valor é uma entrada do Simulink. Como a caixa de marchas é um conjunto reduções, o princípio de conservação da energia mecânica também é aplicado nela.

\subsection{Blocos do controlador e Regeneração}

O último bloco desenvolvido é o do controlador do motor. A sua entrada é a referência de velocidade vinda do acelerador, e a sua saída é o ciclo de traba- 
lho do conversor $\mathrm{CC}$, que é proporcional à tensão eficaz sobre a armadura do motor. Optou-se por um controle em cascata, com dois PI e com realimentação de velocidade e de corrente (figura 5. Isso foi feito, pois o controle em cascata produz sobrepicos de correntes menores do que o controle PID convencional, de acordo com Castrucci (2011). A adoção do método de controle em cascata, por consequência, ajuda a proteger o motor, aumentando sua vida útil.

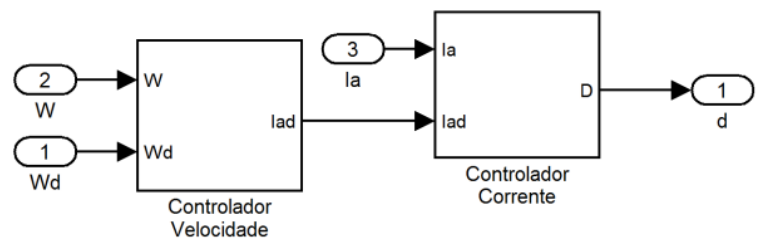

Figura 5. Controlador em cascata.

O controlador de velocidade (Figura 6) é um PI. O valor de saída é o valor de referência para corrente do motor. Essa saída é limitada entre dois patamares, por meio de um mecanismo de saturação.

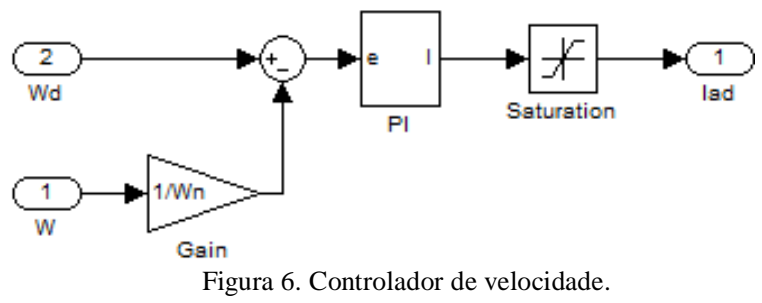

O limite superior é responsável pela proteção do motor, por determinar a máxima corrente permitida quando a máquina elétrica está funcionando como motor.

O limite inferior determina a ocorrência ou não de frenagem regenerativa. Se for zero, a corrente nunca inverterá de sentido, não havendo retorno de energia à fonte. Se for um valor negativo, haverá possibilidade de regeneração, com corrente constante em um determinado valor. Este valor limita o máximo valor de corrente durante a regeneração, o que também protege o motor.

A figura 7 mostra o esquema do controlador de corrente implementado.

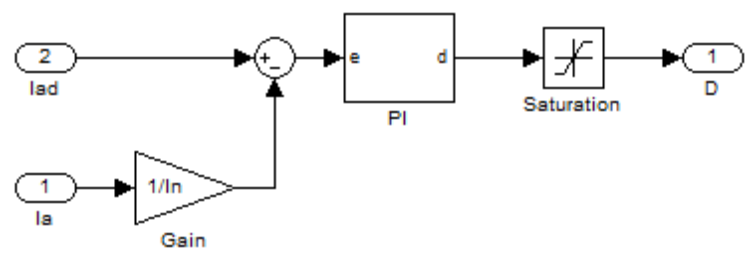

Figura 7. Controlador de corrente.

Como pode ser observado na figura 7, a saída do controlador de velocidade é ligada à referência do controlador de corrente. Este é mais um controlador PI, cuja saída, que varia entre 0 e 1 , controla a tensão de saída do conversor CC.
Ressalta-se que no controlador as grandezas medidas foram normalizadas. Assim, divide-se a velocidade medida pela velocidade nominal do motor, e a corrente medida pela corrente nominal do motor, que foi obtida através de simulação do motor em condições nominais de operação. Os valores dos limites de saturação, por consequência, também são adimensionais.

\section{Resultados e Discussões}

\subsection{Parâmetros Utilizados}

Realizou-se a simulação de um veículo com carroceria e bateria do Nissan Leaf (características do veículo na Tabela 1 , e da bateria na Tabela 2 , com base em Hayes (2011), Nissan (2009) e Nissan (2011), porém com um motor CC de campo bobinado de 100 HP e velocidade base de 1750 RPM (cujas características elétricas e mecânicas estão na Tabela 3 ), em lugar do motor AC síncrono original. O controlador foi ajustado para limitar a corrente em seu valor nominal, de $162,8 \mathrm{~A}$.

Tabela 1. Características do Veículo.

\begin{tabular}{|l|l|}
\hline \multicolumn{1}{|c|}{ Parâmetros } & \multicolumn{1}{c|}{ Valor } \\
\hline Peso bruto $(\mathrm{kg})$ & 1560 \\
\hline Área frontal $\left(\mathrm{m}^{2}\right)$ & 2.29 \\
\hline Coeficiente aerodinâmico (adimensional) & 0.28 \\
\hline Raio das rodas $(\mathrm{m})$ & 0.3 \\
\hline Distância do eixo dianteiro ao CG $(\mathrm{m})$ & 1.54 \\
\hline Distância do eixo traseiro ao CG $(\mathrm{m})$ & 1.535 \\
\hline Altura do CG $(\mathrm{m})$ & 1.0 \\
\hline
\end{tabular}

Tabela 2. Características da Bateria.

\begin{tabular}{|l|l|}
\hline \multicolumn{1}{|c|}{ Parâmetros } & \multicolumn{1}{c|}{ Valor } \\
\hline Tipo & Íon de lítio \\
\hline Tensão nominal total (V) & 345 \\
\hline Número de baterias em série & 4 \\
\hline Número de ramos em paralelo & 48 \\
\hline Carga total (Ah) & 69.6 \\
\hline Estado inicial (\%) & 100 \\
\hline
\end{tabular}

\subsection{Simulações Efetuadas}

Primeiramente fez-se necessária a escolha de uma transmissão adequada para o conjunto veículo/motor para melhor desempenho do conjunto. Para tanto, realizou-se os seguintes procedimentos: Simulou-se o conjunto veículo/motor CC acoplado por duas diferentes transmissões, uma fixa e uma manual, para comparação entre elas.

Comparou-se a aceleração deste veículo de 0 a $100 \mathrm{~km} / \mathrm{h}$, quando este possuía cada uma das transmissões. 
Tabela 3. Parâmetros do Motor CC.

\begin{tabular}{|l|l|}
\hline \multicolumn{1}{|c|}{ Parâmetros } & \multicolumn{1}{c|}{ Valor } \\
\hline Resistência de armadura $(\Omega)$ & 0.1968 \\
\hline Indutância de armadura $(\mathrm{H})$ & 0.003442 \\
\hline Resistência de campo $(\Omega)$ & 58.82 \\
\hline Indutância de campo $(\mathrm{H})$ & 7.267 \\
\hline Indutância mútua $(\mathrm{H})$ & 0.5003 \\
\hline Coeficiente de atrito seco $(\mathrm{Nm})$ & 10.58 \\
\hline Coeficiente de atrito viscoso $(\mathrm{Nm} . \mathrm{s} / \mathrm{rad})$ & 0.01156 \\
\hline Momento de inércia (kg.m²) & 0.4089 \\
\hline Indutância de amortecimento $(\mathrm{H})$ & 0.005 \\
\hline $\begin{array}{l}\text { Tensão máxima do conversor de armadura } \\
\text { (V) }\end{array}$ & 500 \\
\hline Tensão do conversor de campo (V) & 300 \\
\hline
\end{tabular}

Ambas foram dimensionadas de forma que o carro tivesse velocidade máxima de $150 \mathrm{~km} / \mathrm{h}$, como o carro real. Para tanto, utilizou-se a equação (1):

$$
\text { Gear }=\frac{\frac{V_{M A X}}{R_{\text {WHEEL }}}}{\omega_{M A X}}
$$

Nesta equação, $\mathrm{V}_{\text {MAX }}$ é máxima velocidade desejada do veículo, $R_{\text {WHEEL }}$ é o raio das rodas, e $\omega_{\text {MAX }}$ é a máxima velocidade angular do motor. $\mathrm{O}$ valor obtido corresponde ao valor da redução da transmissão fixa e ao valor da redução da última marcha da caixa manual. Os valores usados de redução em cada caso estão na Tabela 4.

Tabela 4. Valores de Redução Utilizados.

\begin{tabular}{|l|l|}
\hline \multicolumn{1}{|c|}{ Reduções (adimensional) } & Valor \\
\hline Transmissão fixa & 1.3195 \\
\hline Caixa de marchas: primeira marcha & 5.0000 \\
\hline Caixa de marchas: segunda marcha & 3.0000 \\
\hline Caixa de marchas: terceira marcha & 2.0000 \\
\hline Caixa de marchas: quarta marcha & 1.3195 \\
\hline
\end{tabular}

As velocidades em que ocorreu cada troca de marcha estão na Tabela 5. Para tal, mediu-se qual a velocidade máxima desenvolvida por cada redução, e efetuou-se a troca de marcha antes que ela fosse atingida.

Tabela 5. Velocidades para a troca de marcha.

\begin{tabular}{|l|l|}
\hline \multicolumn{1}{|c|}{ Troca de marcha } & Valor $(\mathrm{km} / \mathrm{h})$ \\
\hline Primeira para a segunda & 25 \\
\hline Segunda para a terceira & 42 \\
\hline Terceira para a quarta & 65 \\
\hline
\end{tabular}

Mediu-se a velocidade do veículo (Figura 8), em cada caso:

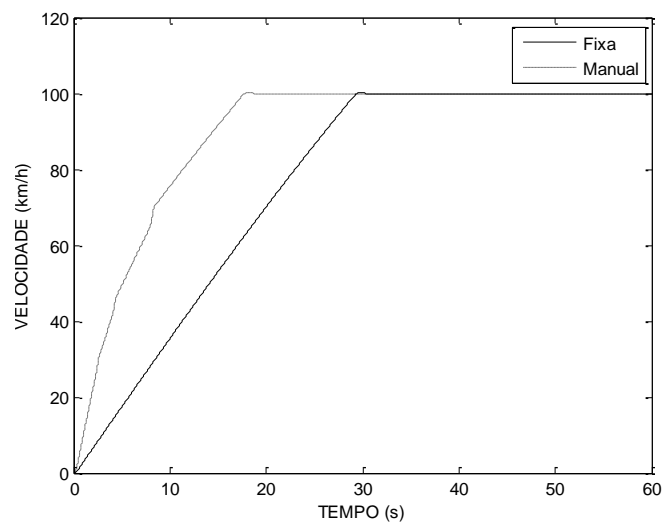

Figura 8. Velocidade do veículo.

Dos gráficos acima, pode-se perceber que o veículo com transmissão manual desenvolveu um melhor perfil de aceleração, chegando à velocidade final 10 segundos mais rápido que o carro com transmissão fixa.

Mediu-se também a corrente elétrica consumida pelo motor me cada caso (Figura 9):

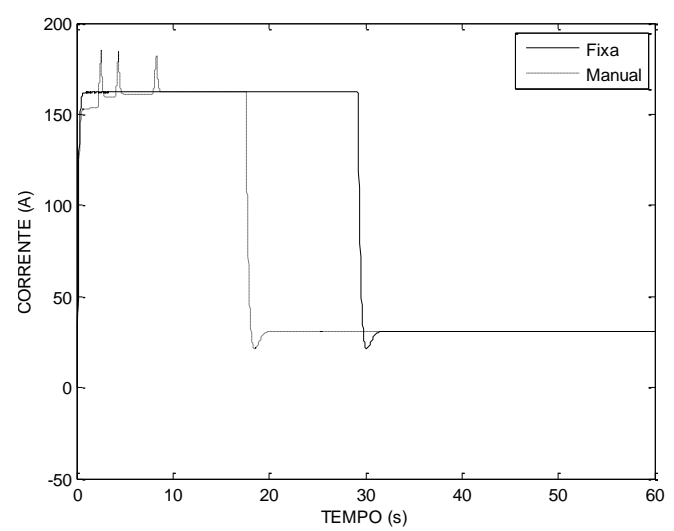

Figura 9. Corrente elétrica consumida pelo veículo.

Na Figura 9, vê-se que em ambos os casos o controlador manteve a corrente consumida pelo motor em seu valor nominal (no caso, 162,8 A), e que o segundo caso nas trocas de marcha apresentava picos mínimos de potência.

Outra variável analisada foi o estado de carga da bateria (Figura 10).

Nela, vê-se que o estado de carga final dos veículos foi igual, porque o carro com transmissão manual desenvolveu uma maior aceleração, o que demanda mais energia, mas chegou mais rápido à velocidade final, o que faz com que o total consumido se mantenha constante. Assim, o melhor desempenho mostrado anteriormente da transmissão manual não compromete o estado de carga da bateria.

Pode-se concluir pela análise dos gráficos que a transmissão manual mostrou-se superior à transmissão fixa na aceleração do veículo de forma considerável, enquanto que a transmissão fixa não proporcionou nenhum pico de corrente no motor. No entan- 
to, o motor CC é capaz de suportar os picos de corrente visualizados coma transmissão manual (eles foram minimizados pelo controlador). Desse modo, escolheu-se a caixa manual de marchas para o conjunto veículo/motor CC.

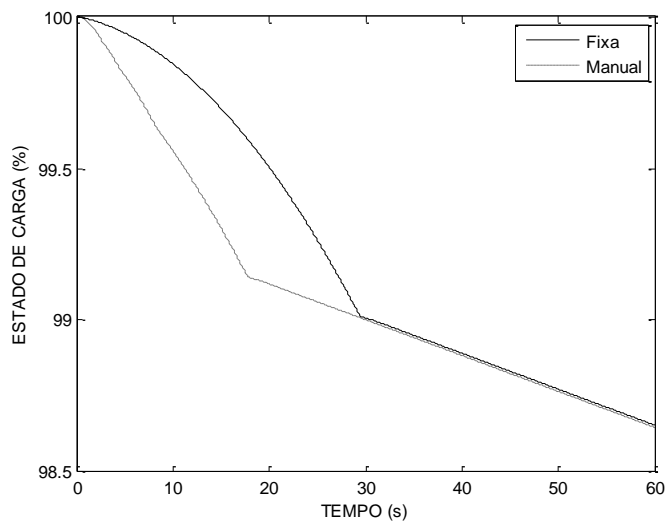

Figura 10. Estado de carga da bateria.

Para avaliar o funcionamento da frenagem regenerativa realizaram-se simulações em pista plana do veículo proposto com e sem a frenagem elétrica. Simulou-se por $60 \mathrm{~s}$, de forma que aos 25 segundos, a referência de velocidade passava de $100 \mathrm{~km} / \mathrm{h}$ para $0 \mathrm{~km} / \mathrm{h}$ (parada total do veículo).

Também se mediu a velocidade do veículo (Figura 11):

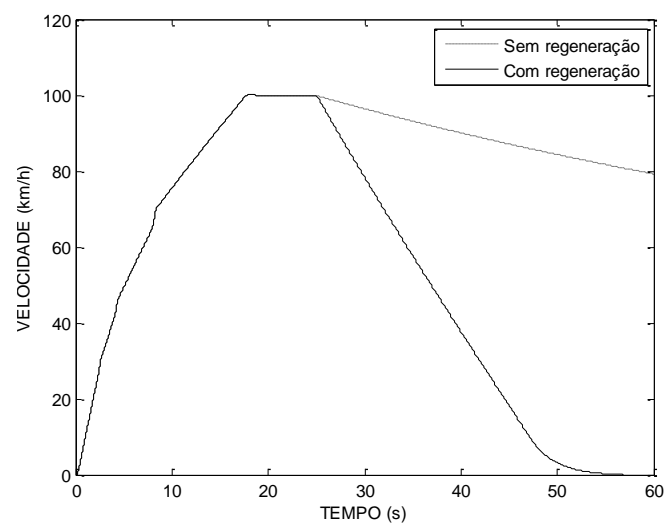

Figura 11. Velocidade do veículo, sem e com regeneração.

Vê-se que ocorreu frenagem do veículo, pois a diminuição de velocidade com regeneração foi muito mais acentuada do que o caso original. Ressalta-se que ainda há a necessidade haver um freio mecânico, para segurança dos passageiros, pois a frenagem regenerativa não produz sozinha a frenagem necessária.

Também se mediu a corrente elétrica consumida pelo motor CC (Figura 12).

A Figura 12 mostra o caráter regenerativo do processo: enquanto o veículo tinha sua velocidade reduzida, uma corrente elétrica era devolvida à fonte. Também se vê que o seu valor foi devidamente limi- tado pelo controlador, deforma a proteger-se o motor durante a regeneração.

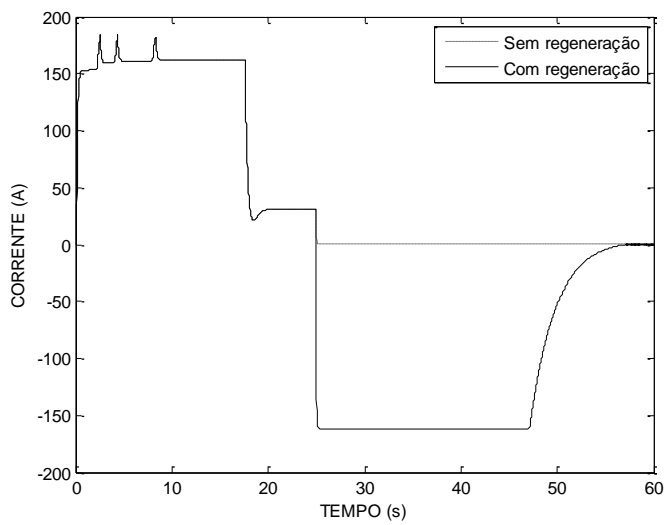

Figura 12. Corrente elétrica consumida pelo veículo, sem e com regeneração.

Por último, mediu-se o estado de carga do banco de baterias, com e sem regeneração de energia:

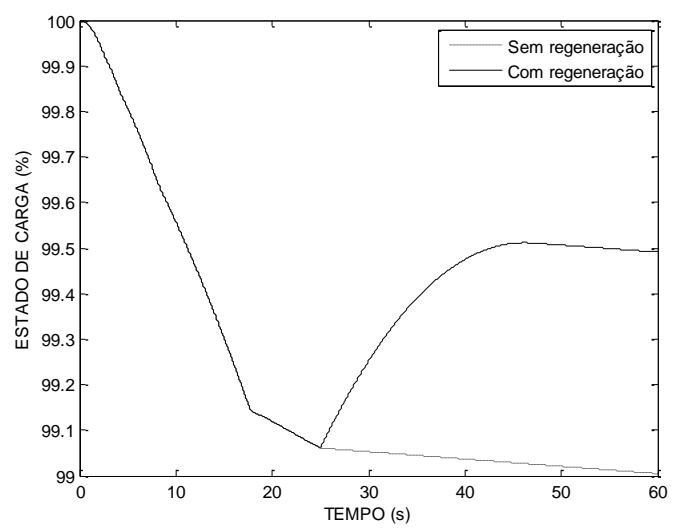

Figura 13. Estado de carga da bateria, sem e com regeneração.

Nesta figura, mostra-se a utilidade da frenagem regenerativa, pois se vê o aumento do estado de carga da bateria durante a regeneração. Nesta simulação, a energia foi totalmente devolvida ao banco de baterias. No entanto, pode-se alocá-la em um banco de ultra-capacitores, para uso posterior na aceleração, pois estes possuem maior densidade de potência do que as baterias.

Desse modo, define-se que a melhor configuração do veículo com motor CC dentre as analisadas é com uma transmissão manual e com implementação da frenagem regenerativa do motor.

\section{Conclusão}

Assim, vê-se que se conseguiu realizar o controle de velocidade do motor, ao mesmo tempo em que se controlou a sua corrente, de forma a protegêlo contra soprepicos elevados de corrente elétrica, o que é danoso aos condutores do motor. Também se mostram com estas simulações duas utilidades da 
ferramenta de simulação desenvolvida: comparar topologias do veículo elétrico (a transmissão, no caso) e analisar os efeitos da implementação da frenagem regenerativa no veículo, tanto os efeitos mecânicos da frenagem do veículo quanto os efeitos elétricos da regeneração de energia.

\section{Agradecimentos}

Agradecimentos ao CNPq e à UFSCar, pelo apoio e pelo financiamento por meio do programa PIBIC-UFSCar.

\section{Referências Bibliográficas}

Castrucci, P. de L., Bittar, A. and Sales, R. M. (2011). Controle Automático. Rio de janeiro, LTC. p. 272 to 278.

Guazzelli, P. R. U, De Francisco, C. A. and Ogashawara, O. (2013). Modelagem e Simulação de Veículo Elétrico. Congresso de Iniciação Científica do Inatel 2013 (Incitel).

Hayes, J. G., Oliveira, R. P. R. de, Vaughan, S. and Egan, M. G. (2011). Simplified Electric Vehicle Power Train Models and Range Estimation. IEEE vehicle Power and propulsion Conference. VPPC'11. p. $1-5$.

Hearn, C. S., Weeks, D. A., Thompson, R. C. and Chen, D. (2010).Electric Vehicle Modeling Utilizing DC Motor Equations. 2010 IEEE/ASME International Conference on Advanced Intelligent Mechatronics. p. 670-675. DOI:

10.1109/AIM.2010.5695785
IEA, (2009). Technology Roadmap: Electric and plug-in hybrid electric vehicles. OECD/IEA, 2009.

Itaipu (2004). Ficha Técnica. Avaiable on: http://www2.itaipu.gov.br/ve/portugues/ficha_te cnica.html. Retrivied 2013-05-21.

Nissan. (2009). Production of $100 \%$ Electric, ZeroEmission Nissan LEAF begins at Oppama, Japan [online]. Avaiable on: http://www.nissanglobal.com/EN/NEWS/2010/_STORY/10102201-e.html. Acessed 14 May 2013.

Nissan. (2011). Nissan Leaf Owner's Manual [online]. Avaiable on: http://large.stanford.edu/courses/2012/ph240/lan dreman 1/docs/2012-Nissan-LEAF.pdf. Acessed 10 May 2013.

Njoya, S., Tremblay, O. and Dessaint, L.A. (2009). A Generic Fuel Cell Model for the Simulation of Fuel Cell Vehicles. IEEE vehicle Power and propulsion Conference. VPPC '09. p. 1722 1729.

Sefik, I. and Hiyama, T. (2011). Performance Evaluation of Hybrid powertrain system Simulation Model for Toyota Prius Car. 2011 International Aegean Conference on Electrical Machines and Power Eletronics and 2011 Electromotion Joint Conference (ACEMP). p. 404 - 407. DOI: 10.1109/ACEMP.2011.6490632

Tesla, (2013). Model S Specs [online]. Avaiable on: http://www.teslamotors.com/models/specs. Acessed 21 May 2013. 